\title{
Changes Caused in the Refractivity and Density of Glass by Annealing
}

\author{
By Arthur Q. Tool, Leroy W. Tilton, and James B. Saunders
}

\begin{abstract}
The changes in the refractivity of several glasses caused by annealing at different temperatures are presented. The results are given in the form of equilibrium temperature coefficients, as the glasses were annealed at each annealing temperature until there was no further change in the refractivity or density. The measurements on refractivity and density were made at standard atmospheric temperatures since the changes in these properties at such temperatures have a greater practical significance than the corresponding changes at temperatures within the annealing range.
\end{abstract}

\section{Introduction}

Investigation has shown that the physical properties of a glass depend not only on its actual temperature, but also on the annealing or heat treatment to which it has been subjected $[1,2,3] .{ }^{1}$ It has also been shown that annealing ordinarily causes a glass to approach a condition of equilibrium at the temperature of the annealing treatment. Moreover, the effect of any annealing treatment on the properties of a glass is a function of the change that the treatment has caused in the equilibrium temperature $[3,4,5]$.

To determine the change caused in any property by a given change in the equilibrium temperature, it is first necessary to establish equilibrium conditions at a number of annealing temperatures. Also, it is necessary in each case, after equilibrium has been established, to cool the glass to some standard temperature for measurement of the coefficient representing the property under investigation.!

Unless the measurements are made at a standard temperature rather than at the temperature of treatment, the results obtained represent effects produced, not only by a change in equilibrium

\footnotetext{
${ }_{1}$ Figures in brackets indicate the literature references at the end of this
} paper. temperature [5], but also by the change in actual temperature. Moreover, the results, even when determined at a standard temperature, will depend on the temperature chosen. This follows because the temperature coefficients of most properties also vary with the equilibrium temperature. For example, it has been shown [6, 7] that both the thermal expansivity and volume of glass increase with the equilibrium temperature. Consequently, the change in volume for a given change in equilibrium temperature is greater when measured at a standard temperature within the annealing range than when measured at atmospheric temperatures.

Refractivity is one of the properties that is changed appreciably, even if the equilibrium temperature is changed by no more than $1 \mathrm{deg}$ C. Any such change in this property of optical glass has considerable practical significance. This is true not only because a difference in the annealing treatments of different portions of glass from the same melt causes the refractivity to vary from portion to portion, but also because unsatisfactory annealing may cause appreciable equilibrium temperature gradients within individual blanks [8,9]. In other words, these temperature gradients can cause appreciable optical inhomogeneity.

From a practical standpoint, interest centers mainly on the variations that annealing treat- 
ments cause in the refractivity at atmospheric temperatures, since optical glass is generally used at such temperatures. Lebedeff and Stozarov $[10,11]$ determined the change in refractivity for several glasses as their equilibrium temperature was changed. However, their measurements of the refractivity were made at the temperatures of treatment. To determine the corresponding indices under atmospheric conditions from such measurements, the average temperature coefficients for the ranges from the treating temperatures to normal atmospheric temperature must be determined for each condition of equilibrium. As these determinations add greatly to the work involved, the measurements at the treating temperatures were not undertaken in procuring the results presented in this or in previous reports $[2,12]$. That is, all refractivity and density determinations were made at standard atmospheric temperatures. Recently, MacMaster [13] has reported the results of a similar investigation in which the measurements of the indices were also made only at a standard atmospheric temperature.

To differentiate between the equilibrium and actual temperatures, these temperatures will be designated as $\tau$ and $T$, respectively. Consequently, the coefficients determined in this investigation are designated as $\Delta n_{D} / \Delta \tau$ and $\left(\Delta n_{F}-\right.$ $\left.\Delta n_{C}\right) / \Delta \tau$, in which $n_{F}, n_{D}$, and $n_{C}$ are the indices for the $F, D$, and $C$ spectral lines respectively. In some cases, the corresponding coefficient, $\Delta D / \Delta \tau$ for the change in density, $D$, was also determined.

\section{Glasses Investigated}

Most of the glasses tested in this investigation were from melts of optical glass produced by the Glass Section of the National Bureau of Standards, and they represent practically all the types made in quantity by the Glass Section prior to 1930 . The six samples from which prisms were made for measurements on both refractivity and density were mostly from glasses ${ }^{2}$ produced after 1920 ,

\footnotetext{
${ }^{2}$ The composition of melt $494 \mathrm{w}$ as determined by analysis. The percentage compositions as given for the other five of these glasses are estimates based on the batch compositions.

Melt 494, $\mathrm{SiO}_{2} 50.55, \mathrm{PbO} 40.14, \mathrm{Al}_{2} \mathrm{O}_{3} 0.65, \mathrm{~K}_{2} \mathrm{O} 5.93, \mathrm{Na}_{2} \mathrm{O} 2.69, \mathrm{Fe}_{2} \mathrm{O}_{3}$ 0.02 ; melt 467, $\mathrm{SiO}_{2} 39.0, \mathrm{PbO} 54.0, \mathrm{~K}_{2} \mathrm{O} 6.5, \mathrm{Na}_{2} \mathrm{O} 0.5$; melt $573, \mathrm{SiO}_{2} 67.5$, $\mathrm{BaO} 2.5, \mathrm{ZnO} 1.0, \mathrm{~K}_{2} \mathrm{O}$ 10.8, $\mathrm{Na}_{2} \mathrm{O} 7.2, \mathrm{~B}_{2} \mathrm{O}_{3}$ 11.0; melt 529, $\mathrm{SiO}_{2} 74.5$, CaO 13.5, $\mathrm{Na}_{2} \mathrm{O} 12.0$; melt 572, $\mathrm{SiO}_{2} 48.0, \mathrm{CaO} 2.0$, $\mathrm{BaO} 27.0, \mathrm{ZnO} 8.0 \quad \mathrm{~B}_{2} \mathrm{O}_{3} 5.5$, $\mathrm{K}_{2} \mathrm{O} 7.5, \mathrm{Na}_{2} \mathrm{O} 2.0$; melt $480, \mathrm{SiO}_{2} 34.5, \mathrm{BaO} 40.7, \mathrm{ZnO} 7.7, \mathrm{~B}_{2} \mathrm{O}_{3} 10.6, \mathrm{Al}_{2} \mathrm{O}_{3}$ 6.5 .
}

and the prisms were cut from large selected pieces that, in all cases, were large fragments that resulted when the pots of glass were broken open.

The six selected pieces, before being heat treated, were investigated carefully to determine the magnitude of the refractivity gradients in them. It was found [14] that these gradients ranged from $0.6 \times 10^{-6}$ to $4.9 \times 10^{-6} / \mathrm{cm}$, and it is believed that they were chiefly the result of gradients in the equilibrium temperature rather than of variations caused by chemical inhomogeneities or residual elastic strains. Strain was an improbable source of the gradients because the double refraction effects in the large pieces were no greater than they often are in pieces of equal size that have supposedly received adequate annealing, and the effects in the small test samples, that were cut from the large pieces, were presumably negligible. Variations in chemical composition are a possible cause of such gradients as variations of that kind are often the cause of serious optical inhomogeneity; but there is reason to believe that the effect of variations in the chemical composition of the best selected optical glass is very small [15]. Variations in the equilibrium temperature, on the other hand, seem to be a very likely cause of the observed refractivity gradients because $\tau$-gradients, like residual elastic strains, cannot be completely eliminated and also because the refractivity is comparatively very sensitive to differences in $\tau$. In fact, it seems probable that the effects of $\tau$-gradients will usually mask any refractivity gradients arising from variations in the composition of good pieces of optical glass.

Besides the 6 glasses already discussed, 15 additional glasses were subjected to the heat treatments and refractivity measurements required to establish their equilibrium curves. Of these, 14 were also from melts [16] made by the Bureau's Glass Section, and several of them are among those listed in the table of refractive indices that appears in the Smithsonian Tables [17]. One glass, a heavy flint, was of German make and was cut from a Pulfrich refractometer block.

\section{Measurement of Refractivity and Density}

Of the six glasses on which both refractivity and density were determined, the test prisms were about $40 \mathrm{~mm}$ in length, their faces 12 to $16 \mathrm{~mm}$ 
in width, and their refracting angles approximated 60 degrees. Such slender prisms were used to increase the volume for density measurements without making it necessary to reduce the rate of safe cooling after the heat treatments. The average prism of the 14 other Bureau glasses also possessed a refracting angle of about 60 degrees, but its faces were approximately $15 \mathrm{~mm}$ square. The prisms of the German glass were much smaller.

A spectrometer was used in measuring the indices of refraction by the minimum deviation method, and the necessary precautions [18] were taken to obtain high precision. Never less than the usual three spectral lines $(C, D$, and $F)$ were used and, when the dispersion was sufficient, the $D_{1}$ line of the sodium doublet was chosen. Repeated measurements on freshly surfaced prisms indicated that variations in the results on any given prism in an unchanged equilibrium condition were small in the sixth decimal place. However, the lack of precision was considerably greater in the intermediate determinations, which were often made without resurfacing the prisms and merely for observing whether equilibrium was almost reached. This lack of precision in the intermediate observations was caused by surface warping and was particularly troublesome after treatments at the higher temperatures, and also after treatments at the lower annealing temperatures if these treatments immediately followed high-temperature treatments.

The density measurements were made by the Bureau's Capacity and Density Section. The usual displacement method of determining volumes was employed, and the submersing liquid was a suitable kerosine held at $20^{\circ} \mathrm{C}$. Precautions were taken to minimize the errors caused by the action of surface tension on the suspension wire. Several repetitions of the measurements on the samples after each treatment indicated that the maximum variation in the results on any prism of unchanged equilibrium approximated \pm 0.0004 under the worst conditions (comparatively high densities and small volumes).

\section{Heat Treatments}

Small tubular electric furnaces (tubes about $50 \mathrm{~cm}$ in length and $4 \mathrm{~cm}$ in diameter with stoppered ends) were used to treat the glass prisms. To make the temperature distribution within the central third of a furnace more nearly uniform, a heavy-walled metal tube about $15 \mathrm{~cm}$ in length was fitted loosely within the refractory tube of the furnace and midway between its ends. The ends of the metal tube were blocked by diaphragms. The junction of a Pt-Pt-Rh thermocouple was placed near the top and midway between the ends of this enclosed space, and the wires of the couple were led through the rear diaphragm and stopper to the cold-junction box. A thin-walled metal capsule containing the glass to be treated was placed just beneath the hot junction. The glass prism within this capsule rested on a thin sheet of burned asbestos to prevent contact with the metal. This arrangement made rapid cooling and heating without breakage possible.

The furnace was brought to a chosen treating temperature before the glass was introduced. Consequently, the glass was heated to this temperature very quickly. After a treatment of the desired duration was completed at any temperature, the glass, still within the capsule, was removed from the furnace and cooled rapidly in air. Except at the highest treating temperatures employed, this procedure allowed very little change in equilibrium temperature during the heating and cooling.

Samples of the same glass were always treated in the same furnace because it was found that the equilibrium temperatures reached in the various furnaces differed by a few degrees, although the treating temperatures as determined by thermocouples were practically identical. This experience indicated errors in the temperature determinations and, presumably, these errors resulted from peculiarities in the furnaces and thermocouples. Generally, the difference between the equilibrium temperatures attained in two furnaces that were supposedly at the same treating temperature did not change materially even when treatments were made at several well-separated temperatures in the annealing range. Thus, it appears that the error in the interval between any two treating temperatures of a glass was always relatively small because the same furnace was used in both cases. It is estimated that the errors in such interval determinations were between \pm 2 degrees, whereas the maximum errors in the treating temperatures may have been two or three times as great.

The period of treatment required to establish 
equilibrium at low annealing temperatures often exceeded 2 months, while no more than a day was necessary at the highest temperatures used. In many cases, the treatment of a sample at a temperature was repeated one or more times before subjecting the sample to treatment at some other temperature. This repetition of a treatment is one means of determining if an equilibrium condition has been approximated. However, more dependence was placed on the results obtained when it appeared that practically the same equilibrium refractivity or density was reached by increasing it in one case and by decreasing it in another through treatments at the same temperature. The most satisfactory method of accomplishing this opposite approach to an equilibrium condition is the simultaneous treatment of two samples at an intermediate temperature after one has been treated at a higher and the other at a lower temperature.

\section{Experimental Results and Discussion}

Excepting the highest and lowest treating temperatures employed for any glass, all others were generally within the portions of the annealing ranges that are recorded in tables 1 and 2. Usually, the interval between the chosen treating tem- peratures was 10 deg $\mathrm{C}$ and, as previously indicated, the glasses were made to approach equilibrium at the selected points from both undercooled and superheated conditions. At low-treating temperatures, complete agreement between the results attained by these opposed approaches was difficult to approximate as reducing the interval between the results for the refractivity to 1 in the fourth decimal place usually required treatments of 1 or more months' duration for each direction of approach. At high-treating temperatures, closure of the interval was approximated much more easily because the rates of approaching equilibrium are so much higher than at temperatures in the lower part of the annealing range. In fact, equilibrium is often approached so closely in a comparatively short time that the results show an apparent overlap instead of a gap if the treating temperature is not carefully controlled. In other words, the refractivity measurements after treatments of adequate duration can show an apparent overlapping of 1 in the fourth decimal place if an error of -2 degrees is made in the treating temperature of the undercooled sample, and an equal, but opposite, error is made in the case of the superheated sample. Two samples in different initial conditions were often treated simultaneously in order to prevent such overlapping.

TABLE 1.-The equilibrium-temperature coefficients of refractivity and density for six glasses

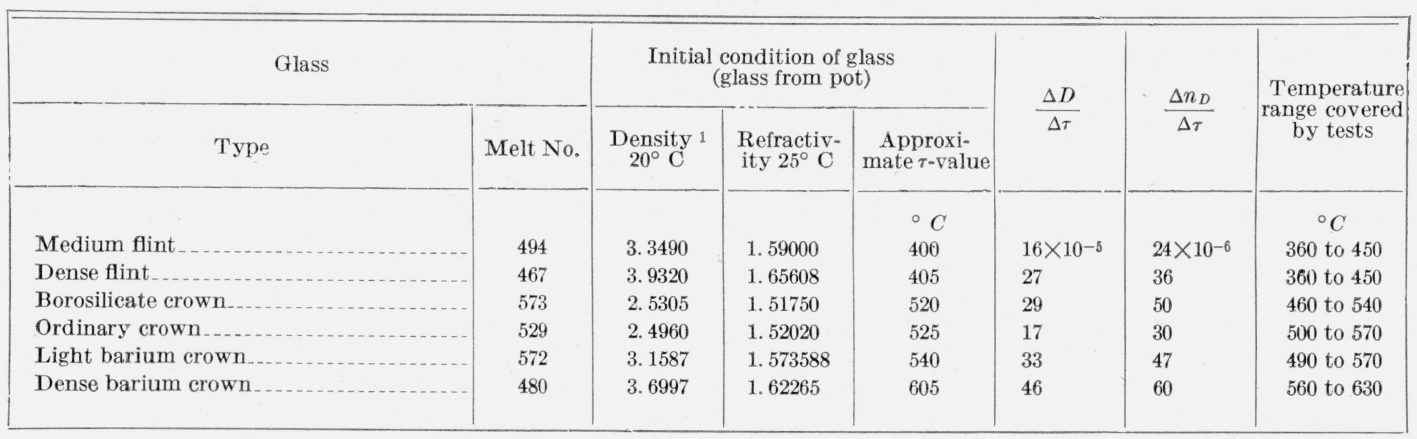

${ }_{1}$ The densities, D, were determined by E. L. Peffer and E. E. Hill of the Capacity and Density Section of the National Bureau of Standards. 
TABLE 2.-Equilibrium-temperature coefficients of refractivity for 15 additional glasses

\begin{tabular}{|c|c|c|c|c|c|c|}
\hline \multicolumn{2}{|l|}{ Glass } & \multicolumn{2}{|c|}{ Initial condition } & \multirow{2}{*}{$\frac{\Delta n_{D}}{\Delta \tau}$} & \multirow{2}{*}{$\frac{\Delta\left(n_{F}-n_{C}\right)}{\Delta \tau}$} & \multirow{2}{*}{$\begin{array}{l}\text { Temperature } \\
\text { range cover- } \\
\text { ed by tests }\end{array}$} \\
\hline Type & Melt No. & $n_{\mathrm{D}}$ at $25^{\circ} \mathrm{C}$ & $\tau$-value & & & \\
\hline & . & & ${ }^{\circ} \mathrm{C}$ & & & ${ }^{\circ} \mathrm{C}$ \\
\hline Light erown ....... & 20 & 1. 51780 & 464 & $27 \times 10^{-6}$ & $+0.6 \times 10^{-7}$ & 410 to 510 \\
\hline Do._... & 103 & 1. 51820 & 448 & 30 & -1.2 & 410 to 480 \\
\hline Do ${ }^{a}$ & 123 & 1. 51714 & - & -.. & . & \\
\hline Borosilicate crown ...... & 94 & 1. 52002 & 510 & 38 & -0.7 & 480 to 540 \\
\hline Do a & 241 & 1. 52430 & 519 & 53 & -1.6 & 480 to 540 \\
\hline Light barium crown & 87 & 1. 57340 & 541 & 42 & -4.6 & 510 to 560 \\
\hline Do a & 116 & 1. 57406 & 539 & 41 & -4.6 & 510 to 570 \\
\hline Dense barium crown a & 151 & 1. 61786 & 579 & 54 & -0.0 & 540 to 620 \\
\hline Barium flint a & 135 & 1. 56819 & 495 & 36 & .0 & 460 to 530 \\
\hline Do & 145 & 1. 55239 & 493 & 31 & 0 & 460 to 530 \\
\hline Light flint . . . . . . & 33 & 1. 58484 & 463 & 23 & +2.3 & 420 to 490 \\
\hline Do s & 188 & 1. 58038 & 455 & 25 & +1.4 & 420 to 480 \\
\hline Medium flint ...................... & 110 & 1. 62493 & 426 & 28 & +3.0 & 380 to 470 \\
\hline Do a & 163 & 1. 62491 & 430 & 27 & +3.9 & 390 to 460 \\
\hline Dense flint $\mathrm{s}^{\mathrm{a}} \ldots$ & 76 & 1. 65548 & 439 & 28 & +5.3 & 390 to 460 \\
\hline Very dense flint $b \ldots$ & . . - & 1. 91794 & 385 & 48 & +40.0 & 340 to 400 \\
\hline
\end{tabular}

a Glasses listed in Smithsonian Tables, Ed. 7, Rp. 3, p. 277 (1927).

b A German product used on Pulfrich refractometers.

Figure 1, which was prepared for a progress report $[12],{ }^{3}$ gives a typical representation of preliminary data obtained by these investigations. Figure 2 shows both refractivity and density data obtained on a third glass. The caret-like symbols indicate the indices reached by treating an undercooled glass at various temperatures for periods of treatment that were not always sufficient, and two or more carets at the same temperature indicate that added treatments were given in order to bring about a closer approach to equilibrium. The inverted carets show the indices reached by treating superheated glass at the temperatures indicated until equilibrium was approximated. The trend of the results for different temperatures is indicated by straight lines, as the equilibrium indices are related almost linearly to the treating temperatures as long as these temperatures are confined to that portion of the annealing range covered by the tests. That is, $d n / d \tau$ is approximately constant and can be determined graphically as indicated by the straight lines, $A$ and $B$, in figure 1 . The data on the changes in density indicate that $d D / d \tau$ also is practically constant in most cases (see fig. 2).

These coefficients, so determined both for

\footnotetext{
${ }^{3}$ The figure was shown when a preliminary paper concerning this investigation was presented at the Corning meeting of the American Optical Society in 1937, and is similar to an earlier graphical representation shown at the Ithaca meeting of the society in 1925 [2], while demonstrating how the refractivity and density of another glass (NBS melt 494) were changed as the equilibrium condition was changed by annealing at different temperatures.
}

density and for refraction of the $D$-line of sodium, are presented in table 1 . It is estimated that the maximum error in coefficients is about \pm 5 percent. Preliminary data on some of these coefficients have been presented in annual reports of the Director of the National Bureau of Standards [19]. Since then, there has been some revision, and data on the initial condition of the glasses is now included. The usual type-designations that are employed in both tables 1 and 2, are not particularly significant because such designations generally have no unique relation to composition, which is a factor that often varies appreciably even in successive melts of any particular type. Consequently, the coefficients of a number of glasses bearing the same type designation often vary over a considerable range.

Excepting the results for a very dense flint, table 2 presents data obtained on earlier melts made at the National Bureau of Standards. The samples used for five of these melts were those on which the indices appearing in the Smithsonian Physical Tables [17] were obtained. Of the other three Bureau melts for which the indices are given in the Smithsonian tables, the samples used for the earlier index determinations were not available. In one of these cases, melt 123, there was also no duplicate sample, but its composition was about the same as that of melt 103. In the other two cases, melts 151 and 163, duplicate samples were available, but their indices differed 


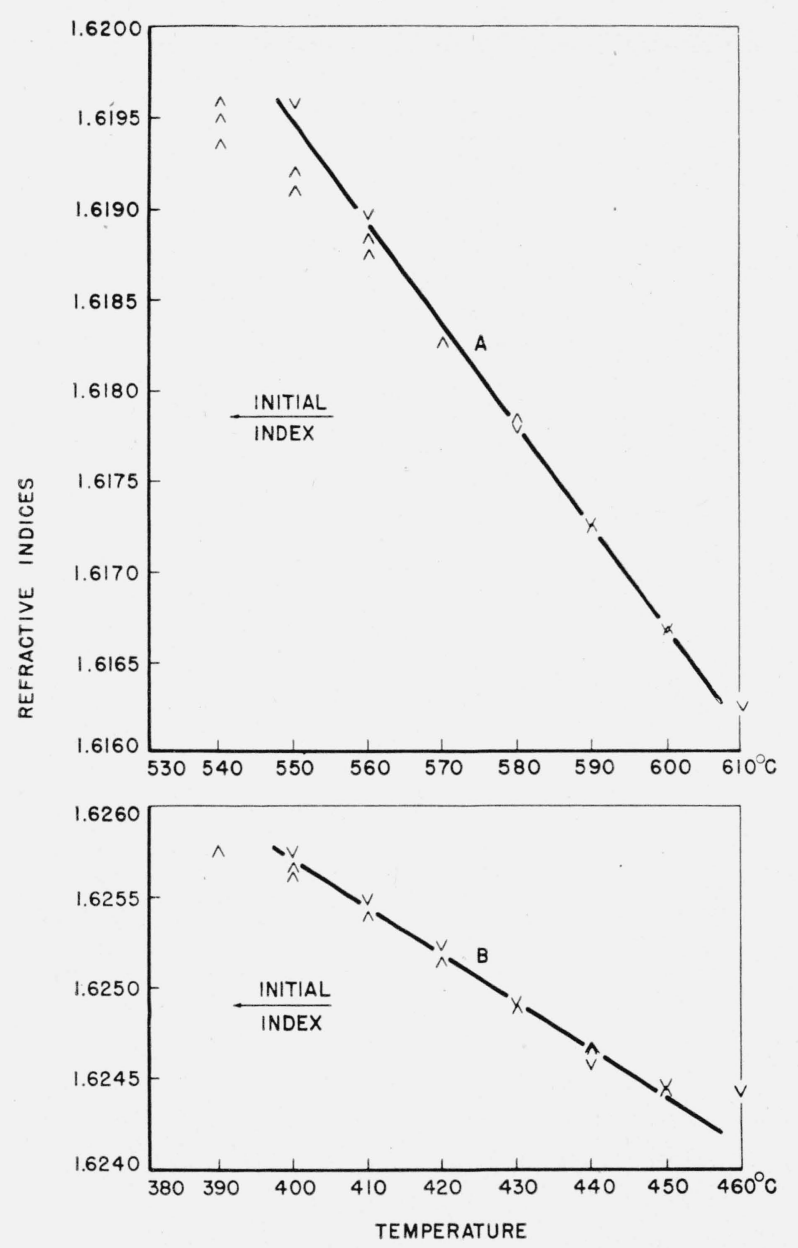

FIGURE 1.-Examples of results obtained on refractivity by annealing at different temperatures until equilibrium was reached.

$A$, results obtained on melt 151 . Carets and inverted carets indicate indices reached as the refractivity was being increased and decreased, respectively, by bringing the glass to equilibrium at the temperatures indicated. Two or more carets at the same temperature signify that observations were made before equilibrium was reached. $B$, Similar results on melt 163 .

from those published in the tables. For melt 151 , the difference $(0.0003)$ possibly results almost entirely from differences in the annealing. For melt 163, the comparatively large difference (0.00234) suggests a possible error in sample numbers.

The maximum errors in the determinations of the treating temperatures and the coefficients, $\Delta n / \Delta \tau$, are thought to be about the same for table 2 as for table 1 . The magnitude of the errors in the determinations of the added dispersion coefficient, $\left(\Delta n_{F}-\Delta n_{C}\right) / \Delta \tau$, are rather uncertain and, in some cases, are probably large; nevertheless they are apparently insufficient to affect the gen-

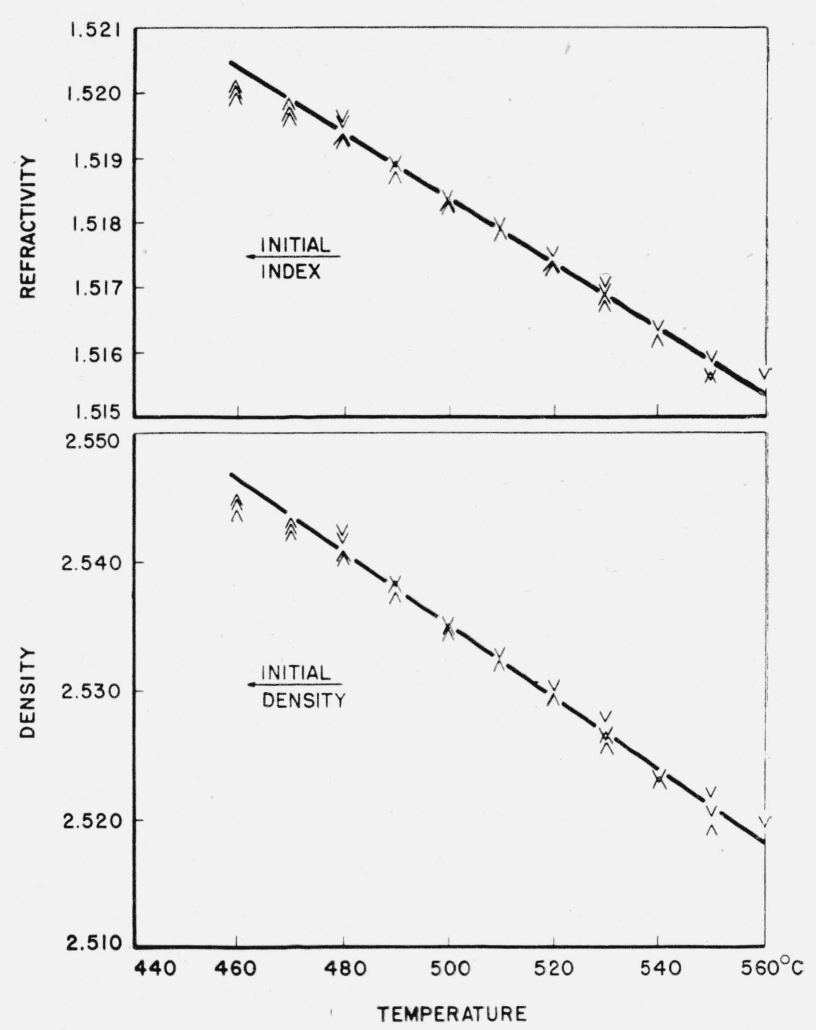

FiguRE 2.-Results obtained on both density and refractivity of melt 573.

eral trend of the results. As would be expected in view of the relatively large values of $\Delta D / \Delta \tau$ as compared to the ordinary expansivities of the glasses, $\Delta n / \Delta \tau$ is always negative, and the dispersion coefficients are either negative or almost zero whenever, as in crown glasses, the ultraviolet absorption band is so remote from the visible spectrum that a shift of this band plays a secondary role compared to that of the density change in causing changes in refractivity even for blue light. However, in the flints in which there is presumably a comparatively pronounced extension or shift of the absorption band or region toward the visible spectrum as $\tau$ increases as well as when $T$ increases, the dispersion coefficients have positive values that are large in dense flints in which the transmission for blue light is reduced appreciably because of the proximity of the absorption region.

The relations of the equilibrium temperature coefficients of refractivity and dispersion to the coefficient $\Delta D / \Delta \tau$ and to the shift of the ultraviolet absorption band as $\tau$ changes are clarified greatly 
by a consideration of Pulfrich's results on the ordinary temperature coefficients of refractivity and dispersion for glasses in the range from $0^{\circ}$ to $100^{\circ} \mathrm{C}$. Pulfrich's results [20] suggest that dispersion coefficients, such as $\Delta\left(n_{F}-n_{C}\right) / \Delta T$, are always positive, although the coefficients $\Delta n / \Delta T$ may be either positive or negative, depending on the kind of glass and the temperature range. Obviously, the decreasing density of a glass as it heats would result in negative temperature coefficients for both refractivity and dispersion if there was no absorption band effect. Moreover, it is well known that the proximity of the ultraviolet absorption region to the visible spectrum considerably increases the refractivity, especially for light near the short wavelength end of the spectrum, and also that the relative increase in refractivity for shorter wavelengths becomes greater as the absorption region moves toward the visible spectrum because of an increasing temperature. Thus, Pulfrich explained his results on the assumption that, as $T$ increases, the negative effects of decreasing density are opposed by the positive effects caused by the proximity of the ultraviolet absorption region to the visible spectrum and by the shift of this region toward longer wavelengths as $T$ increases.

According to this explanation, the effects attributed to the absorption region and its shift with temperature generally overshadow those caused by the change in density. Ordinarily, the ultraviolet region is nearer the visible spectrum in flints than in crowns, and it actually encroaches upon it in some cases. As a result, the temperature coefficients of dispersion and refractivity are positive in flints and generally larger than in crowns. Although smaller, those of the latter glasses are, with some exceptions, such as the negative coefficients of refractivity found by Pulfrich, also positive. That all of the glasses tested in the present investigation have negative equilibrum temperature coefficients, such as $\Delta n_{D} / \Delta \tau$, and that many of the crowns also have negative dispersion coefficients, such as $\Delta\left(n_{F}-n_{C}\right) / \Delta \tau$, must be ascribed to the fact that $\Delta D / \Delta \tau$ is usually much larger than $\Delta D / \Delta T$, and also to the probability that changes in $\tau$ cause smaller shifts in the ultraviolet absorption region than are caused by equal changes in $T$, even if the latter changes are confined to the range between $0^{\circ}$ and $100^{\circ} \mathrm{C}$.

\section{Summary and Conclusions}

Results for the equilibrium temperature coefficients of refractive index, dispersion, and density were determined for several different optical glasses.

The range of equilibrium temperatures covered was, on the average, about $70^{\circ} \mathrm{C}$ for each glass, and this range comprised most of the annealing range that is usable for optical glasses.

Excepting at the end temperatures of this range, the equilibrium temperature was approached both from above and below and, within the errors of measurement, the same results were obtained in both cases.

The time required to establish equilibrium ranged from several months at low annealing temperatures to only a few hours at the highest employed.

To have employed higher temperatures would have required much smaller samples because the cooling from such temperatures must be very rapid if a downward drift of the equilibrium temperature is to be prevented. Such very small samples necessitate the use of less accurate methods for refractivity and density measurements.

By cooling to normal (atmospheric) standard temperatures, the results obtained have a more practical significance than those made at higher temperatures, because it is in the range of atmospheric temperatures that optical glass is ordinarily used.

From the results obtained, it was concluded that the density and the refractivity for any wavelength of light increase almost linearly as the equilibrium temperature decreases; therefore, the equilibrium temperature coefficients of both density and refractivity are negative and practically constant.

The average magnitudes of the equilibrium temperature coefficients of density, and of refractivity for the $D$-line, are about $28 \times 10^{-5}$ and $37 \times 10^{-6} / \mathrm{deg} \mathrm{C}$, respectively, and both are large enough to cause undesirable inhomogeneity even if the gradients in this temperature exceed no more than a tenth of a degree per centimeter in any glass of which exceptional homogeneity is required.

From the results obtained, it appears that the equilibrium temperature coefficients of dispersion 
ure usually negative in crown glasses and positive in flint glasses. This difference in the dispersion coefficients doubtless is related to the location of the ultraviolet absorption band which, in heavy flints, often encroaches on the visible spectrum.

\section{References}

[1] A. Q. Tool and E. E. Hill, Trans. Soc. Glass Tech. 9, 185 (1925).

[2] A. Q. Tool, L. W. Tilton, and E. E. Hill, Am. Optical Soc. \& Rev. Sci. Instr. 12, 490 (1926).

[3] A. Q. Tool and C. G. Eichlin, Am. Ceram. Soc. 14, 276 (1931).

[4] A. Q. Tool, J. Research NBS 34, 199 (1945) RP1637.

[5] A. Q. Tool. J. Research NBS 37, 73 (1946) RP1730.

[6] J. B. Saunders and A. Q. Tool, BS J. Research 11, 799 (1933) RP626.

[7] A. Q. Tool, D. B. Lloyd, and G. E. Merritt, Am. Ceram. Soc. 13, 632 (1930).

[8] L. W. Tilton, A. N. Finn. and A. Q. Tool, J. Am. Ceram. Soc. 11, 292 (1928).
[9] L. W. Tilton, A. N. Finn, and A. Q. Tool, BS Sci. Pap. 22, 719 (1928) S572.

[10] A. A. Lebodeff, Rev. Opt. 5, 1 (1926).

[11] A. I. Stozarov, Trans. Opt. Inst. (Leningrad) 4, 1 (1928).

[12] A. Q. Tool, L. W. Tilton, and J. B. Saunders, J. Am. Opt. Soc. 27, 222 (1937).

[13] H. A. MeMaster, J. Am. Ceram. Soc. 28, 1 (1945).

[14] L. W. Tilton, J. Wash. Acad. Sci. 20, 12 (1930).

[15] F. Eckert, Z. Tech. Phys. 7, 282 (1926).

[16] W. S. Williams and C. C. Rand, J. Am. Ceram. Soc, 2, 422 (1919).

[17] Smithsonian Physical Tables, 7th ed., p. 277 (Smithsonian Institution, Wash., D. C. (1927).

[18] L. W. Tilton, J. Research NBS 14, 393 (1935) RP776.

[19] Annual report of the Director of the Bureau of Standards, Misc. Pub. BS No. 88, p. 21 (1928); Misc. Pub. BS No. 102, p. 25 (1929); Misc. Pub BS No. 115, p. 27 (1930).

[20] C. Pulfrich, Ann. Phys. Chem. 45, 609 (1892).

Washington, December 20, 1946. 